\title{
A Fast and Compact Quantum Random Number Generator
}

\author{
Thomas Jennewein, Ulrich Achleitner†, Gregor Weihs, \\ Harald Weinfurterf, and Anton Zeilinger \\ Institut für Experimentalphysik, Universität Wien, \\ Boltzmanngasse 5, A-1090 Wien, Austria \\ $\dagger$ Institut für Experimentelle Anaesthesie, Universitätsklinik für Anaesthesie und Intensivmedizin, \\ Anichstraße 35, A-6020 Innsbruck, Austria \\ $\ddagger$ Sektion Physik, Ludwig-Maximilians-Universität Muenchen \\ Schellingstr. 4/III D-80799 München, Germany
}

(February 1, 2008)

\begin{abstract}
We present the realization of a physical quantum random number generator based on the process of splitting a beam of photons on a beam splitter, a quantum mechanical source of true randomness. By utilizing either a beam splitter or a polarizing beam splitter, single photon detectors and high speed electronics the presented devices are capable of generating a binary random signal with an autocorrelation time of 11.8 ns and a continuous stream of random numbers at a rate of $1 \mathrm{Mbit} / \mathrm{s}$. The randomness of the generated signals and numbers is shown by running a series of tests upon data samples. The devices described in this paper are built into compact housings and are simple to operate.
\end{abstract}




\section{INTRODUCTION}

Random numbers are a vital ingredient in many applications ranging, to name some examples, from computational methods such as Monte Carlo simulations and programmingt, over to the large field of cryptography for generating of crypto code or masking messages, as far as to commercial applications like lottery games and slot machines2 5 . Recently the range of applications requiring random numbers was extended with the development of quantum cryptography and quantum information processing6. Yet a novelty is the application for which the random number generator presented in this paper was developed for: an experiment regarding the entanglement of two particles, a fundamental concept within quantum theoryd. Firstly this experiment demanded the generation of random signals with an autocorrelation time of $<100 \mathrm{~ns}$. Secondly, for the clarity of the experimental results, it was necessary that true (objective) randomness was implemented.

The range of applications using random numbers has lead both to the development of various random number generators as well as to the means for testing the randomness of their output. Generally there are two approaches of random number generation, the pseudo random generators which rely on algorithms that are implemented on a computing device, and the physical random generators that measure some physical observable expected to behave randomly.

Pseudo random generators are based on algorithms or even a combination of algorithms and have been highly refined in terms of repetition periods $\mathrm{B}\left(2^{800}\right)$ and robustness against tests for randomness 20. But the inherent algorithmic evolution of pseudo random generators is an essential problem in applications requiring unpredictable numbers as the unguessability of the numbers relies on the randomness of the seeding of the internal state. Dependent on the intended application this can be a drawback. The requirements of our specific implementation were even such, that the use of a pseudo random number generator was in itself already ruled out by its deterministic nature.

Physical random generators use the randomness or noise of a physical observable, such as 
noise of electronic devices, signals of microphones, etc. 目. Many such physical sources utilize the behavior of a very large and complex physical systems which have a chaotic, yet at least in principle deterministic, evolution in time. Due to the many unknown parameters of large systems their behavior is taken for true randomness. Still, purely classical systems have a deterministic nature over relevant time scales, and external influences into the random generator may remain hidden.

Current theory implies that the only way to realize a clear and understandable physical source of randomness is the use of elementary quantum mechanical decisions, since in the general understanding the occurrence of each individual result of such a quantum mechanical decision is objectively random (unguessable, unknowable). There exists a range of such elementary decisions which are suitable candidates for a source of randomness. The most obvious process is the decay of radioactive nucleus $\left({ }^{85} \mathrm{Kr},{ }^{60} \mathrm{Co}\right)$ which has already been used 9 . 10 . However, the handling of radioactive substances demands extra precautions, especially at the radioactivity required by the switching rates of our envisaged random signals. Optical processes suitable as a source of randomness are the splitting of single photon beams $\mathbb{1}$, the polarization measurement of single photons, the spatial distribution of laser speckles 12.13 or the light-dark periods of a single trapped ion's resonance fluorescence signal 4 , 司. But only the first two of the mentioned optical processes are fast enough and, in addition, do not require an overwhelming technical effort in their realization. Thus we developed a physical quantum mechanical random generator based on the splitting of a beam of photons with an optical 50:50 beam splitter or by measuring the polarization of single photons with a polarizing beam splitter 11

\section{THEORY OF OPERATION}

The principle of operation of the random generator is shown in Figure 1. For the case of the 50 : 50 beam splitter (BS) (Figure 1(a)), each individual photon coming from the light source and traveling through the beam splitter has, for itself, equal probability to be 
found in either output of the beam splitter. If a polarizing beam splitter (PBS) is used (Figure 1(b)), then each individual photon polarized at $45^{\circ}$ has equal probability to be found in the $\mathrm{H}$ (horizontal) polarization or $\mathrm{V}$ (vertical) polarization output of the polarizer. Anyhow, quantum theory predicts for both cases that the individual "decisions" are truly random and independent of each other. In our devices this feature is implemented by detecting the photons in the two output beams with single photon detectors and combining the detection pulse in a toggle switch $(\mathrm{S})$, which has two states, 0 and 1 . If detector D1 fires, then the switch is flipped to state 0 and left in this state until a detection event in detector D2 occurs, leaving the switch in state 1, until a event in detector D1 happens, and $\mathrm{S}$ is set to state 0 . (Figure 1(c)). In the case that several detections occur in a row in the same detector, then only the first detection will toggle the switch $\mathrm{S}$ into the corresponding state, and the following detections leave the switch unaltered. Consequently, the toggling of the switch between its two states constitutes a binary random signal with the randomness lying in the times of the transitions between the two states. In order to avoid any effects of photon statistic of the source or optical interference onto the behaviour of the random generator the light source should be set to produce $\ll 1$ photon per coherence time.

\section{REALIZATION OF THE DEVICE}

Figure 2 shows the circuit diagram of the physical quantum random generator. The light source is a red light emitting diode (LED) driven by an adjustable current source (AD586 and TL081) with maximally $110 \mu \mathrm{A}$. Due to the very short coherence length of this kind of source $(<1 \mathrm{ps})$ it can be ascertained, that most of the time there are no photons present within the coherence time of the source, thus eliminating effects of source photon statistics or optical interference. The light emerging from the LED is guided through a piece of pipe to the beam splitter, which can be either a 50:50 beam splitter or a polarizing beam splitter. In the latter case the photons are polarized beforehand with polarization foil (POL) at $45^{\circ}$

with respect to the axis of the dual channel polarization analyzer (PBS). The photons in 
the two output beams are detected with fast photo multipliers 1 (PM1, PM2). The PMs are enclosed modules which contain all necessary electronics as well as a generator for the tube voltage, and thus only require a $+12 \mathrm{~V}$ supply. The tube voltages can be adjusted with potentiometers (TV1, TV2) for optimal detection pulse rates and pulse amplitudes . The output signals are amplified in two Becker\&Hickl amplifier modules (A) and transmitted to the signal electronics which is realized in emitter-coupled-logic (ECL). The detector pulses are converted into ECL signals by two comparators (MC1652) in reference to adjustable threshold voltages set by potentiometers, (RV1, RV2). The actual synthesis of the random signal is done within a RS-flip-flop (MC10EL31) as PM1 triggers the S-input and PM2 triggers the $\mathbf{R}$-input of the flip-flop. The output of this flip-flop toggles between the high and low state dependent upon whether the last detection occurred in PM1 or PM2. Finally the random signal is converted from ECL to TTL logic levels (MC10EL22) for further usage.

In order to generate random numbers on a personal computer the signal from the random generator is sampled periodically and accumulated in a 32 bit wide shift register (Figure 3). Every 32 clock cycles the contents of the shift register are transferred in parallel to a personal computer via a fast digital I/O board. In this way a continuous stream of random numbers is transferred to a personal computer.

\section{TESTING THE RANDOMNESS OF THE DEVICE}

Up to now, no general definition of randomness exists and discussions still go on. Two reasonable and widely accepted conditions for the randomness of any binary sequence is its being "chaotic" and "typical". The first of these concepts was introduced by Kolmogorov and deals with the algorithmic complexity of the sequence, while the second originates from Martin-Löv and says that no particular random sequence must have any features that

make it distinguishable from all random sequences 17,18 . With pseudorandom generators it is always possible to predict all of their properties by more or less mathematical effort, due to the fact of knowing their algorithm. Thus one may easily reject their randomness 
from a rigorous point of view. In contrast, the mostly desired feature of a true random generator, its "truth", bears the principal impossibility of ever describing such a generator completely and proving its randomness beyond any doubt. This could only be done by recording its random sequence for an infinite time. One is obviously limited experimentally to finite samples taken out of the infinite random sequence. There are lots of empirical tests, mostly developed in connection with certain Monte Carlo simulation problems, for testing the randomness of such finite samples2 19. The more tests one sample passes, the higher we estimate its randomness. We estimate a test for randomness the better, the smaller or more hidden the regularities may be that it can detect 120.

As the range of tests for the randomness of a sequence is almost unlimited we must find tests which can serve as an appropriate measure of randomness according to the specific requirements of our application. Since the experiment that our random generators are designed for demands random signals at a high rate, we focus on the time the random generators take to establish a random state of its signal starting from a point in time where the output state and the internal state of the generator may be known.

We will briefly describe the relatively intuitive tests that will be applied to data samples taken from the random generator, which we consider to be sufficient in qualifying the device for its use in the experiment.

1. Autocorrelation Time of the Signal: For a binary sequence as produced by our random generator the autocorrelation function exhibits an exponential decay of the form:21

$$
A(\tau)=A_{0} \mathrm{e}^{-2 \mathrm{R}|\tau|},
$$

where $R$ is the average toggle rate of the signal, $A_{0}$ is the normalization constant and $\tau$ is the delay time. Per definition the autocorrelation time is given by

$$
\tau_{a c}=\frac{1}{2 R}
$$


The autocorrelation function is a measure for the average correlation between the signal at a time $t$ and later time $t+\tau$.

2. Internal Delay within the Device: The internal delay time within the device between the emission of a photon and its effect on the output signal. This internal delay time is the minimal time the generator needs to establish a truly random state of its output.

3. Equidistribution of the Signal: This is the most obvious and simple test of randomness of our device, as for random generator the occurrence of each event must be equally probable. Yet, by itself the equidistribution is not a criterion for the randomness of a sequence.

4. Distribution of Time Intervals between Events: The transitions of the signal generated by our system are independent of any preceding events and signals within the device. For such a Poissonian process the time intervals between successive events are distributed exponentially in the following way:

$$
p(T)=p_{0} \mathrm{e}^{-\mathrm{T} / \mathrm{T}_{0}},
$$

where $p(T)$ is the probability of a time interval $T$ between two events, $T_{0}=1 / R$ is the mean time interval and is the reciprocal value of the average toggle rate $R$ defined earlier and $p_{0}$ is the normalization constant. The evaluation of $p(T)$ for a data sample taken from our generator shows directly for which time intervals the independence between events is ascertained and for which time intervals the signal is dominated by bandwidth limits or other deficiencies within the system.

5. Further Illustrative Tests of Randomness: These statistical tests will be applied to samples of random numbers produced by the random generator in order to illustrate the functionality of the device. For the application our random generators are designed for these statistical measures are not as important as the tests described above, and 
the tests proposed here represent just a tiny selection of possible test. Yet, these tests allow a cautious comparison of random numbers produced with our device with random numbers taken from other sources. The code for the evaluation evaluation of these tests was developed in 11 .

(a) Equidistribution and Entropy of $n$-Bit Blocks: Provided that the sample data set is sufficiently long, all possible $n$-bit blocks (where $n$ is the length of the block) should appear with equal probability within the data set. A direct, but insufficient, way of determining the equidistribution of a data set is to evaluate the mean value of all $n$-bit blocks, which should be $\left(2^{n}-1\right) / 2$. This will give the same result for any symmetric distribution. The distribution of $n$-bit blocks of a data set corresponds to the entropy, a value which is often used in the context of random number analysis. The entropy is defined as:

$$
H_{n}=-\sum_{n} p_{i} \log _{2} p_{i}
$$

and is expressed in units of bits. $p_{i}$ is the empirically determined probability for finding the $i$-th block. For a set of random numbers a block of the length $n$ should produce $n$ bits of entropy. In the case of bytes, which are blocks of 8 bits, the entropy of these blocks should be 8 bits.

(b) Blocks of $n$ Zeros or Ones: Another test for the randomness of a set of bits is the counting of blocks of consecutive zeros or ones. Each bit is equally likely a zero as a one, therefore the probability of finding blocks of $n$ concatenated zeros or ones should be proportional to a $2^{-n}$ function.

(c) Monte-Carlo estimation of $\pi$ : A pretty way of demonstrating the quality of a set of random numbers produced by a random generator is a simple Monte Carlo estimation of $\pi$. The idea is to map the numbers onto points on a square with a quarter circle fitted into the square and count the points which lay within the quarter circle. The ratio of the number of points lying in the circle and the total 
number of points is an estimation of $\pi$.

\section{OPERATION OF THE DEVICE}

Two random generators were each built into single width NIM-module (dimensions: $\left.25 * 19 * 3 \mathrm{~cm}^{3}\right)$ in order to match our existing equipment. The optical beam splitter, the two photo multipliers and the pulse amplifiers are mounted on a base-plate within the modules and the electronics is realized on printed circuit boards. The random generator modules require only a standard voltage supply of $\pm 6 \mathrm{~V}$ and $+12 \mathrm{~V}$. The random signal generators were configured either with a 50:50 beam splitter or with a polarizing beam splitter as the source of randomness. In both cases they performed equally well. Yet, the polarization measurement of the photons offers the advantage of adjusting the division ratio of the photons in the two beams by slightly rotating the polarization foil sitting just in front of the beam splitter. The results presented here were all obtained from a random signal generator configured with the polarizing beam splitter.

After warm up the devices require a little adjustment for maximum average toggle rate and equidistribution of the output signal. The average toggle rate of the random signals is checked with a counter and the equidistribution is checked by sampling the signal a couple of thousand times and counting the occurrences of zeros and ones. These measures are both optimized by trimming the reference voltage of the discriminators (RV1, RV2) and by adjusting the tube voltages of the photo multipliers (TV1, TV2). The maximum average toggle rate of the random signals at the output of the random number generators is $34.8 \mathrm{MHz}$. Once the devices are set up in this way they run stably for many hours.

Typically the PMs produce output pulses with an amplitude of maximally $50 \mathrm{mV}$ at a width of 2 ns. The rise and fall time of the signals produced by the random number generators is $3.3 \mathrm{~ns}$. As it turns out, this limit is set by the output driver stage of the electronics. The transition times of the internal ECL signals was measured to be less then $1 \mathrm{~ns}$, which is in accordance with the specifications of this ECL logic. 


\section{PERFORMANCE OF THE DEVICE}

The time delay between the emission of a photon from the light source and its effect on the output signal after running through the detectors and the electronics was measured by using a pulsed light source instead of the continuous LED and observing the electronic signals within the generator on an oscilloscope. The total time delay between a light pulse and its effect on the output was $75 \mathrm{~ns}$, and consists of $20 \mathrm{~ns}$ time delay in the light source, light path and the detection, $20 \mathrm{~ns}$ time delay in the amplifiers and cables and $35 \mathrm{~ns}$ time delay in the main electronics.

In order to evaluate the autocorrelation time of the signal produced by the device, signal traces consisting of 15000 points were recorded on a digital storage oscilloscope for three different average toggle rates. The sampling rate of the oscilloscope was $500 \mathrm{MS} / \mathrm{s}$ for the toggle rates of $34.8 \mathrm{MHz}$ and $26 \mathrm{MHz}$ and $250 \mathrm{MS} / \mathrm{s}$ for the toggle rate of $16 \mathrm{MHz}$. The autocorrelation function for each trace was evaluated on a personal computer and the autocorrelation time $\tau_{A C}$ was extracted by fitting an exponential decay model to these functions. (Figure 4) The resulting autocorrelation times are $11.8 \pm 0.2 \mathrm{~ns}(14.4 \mathrm{~ns})$ for the $34.8 \mathrm{MHz}$ signal, $16.0 \pm 0.6 \mathrm{~ns}(19.2 \mathrm{~ns})$ for the $26 \mathrm{MHz}$ signal and $30.7 \pm 0.5 \mathrm{~ns}(31.3 \mathrm{~ns})$ for the $16 \mathrm{MHz}$ signal which are comparable with the autocorrelation times calculated with expression (2) from the average toggle rate $R$, given in parenthesis.

The time difference between successive toggle events of the random signal is measured with a time interval counter. The start input of the counter is triggered by the positive transition, and the stop input is triggered by the negative transition of the signal. Figure 5 shows the distribution of $10^{6}$ time intervals for a random signal with an average signal toggle rate of $26 \mathrm{MHz}$. For times $<3 \mathrm{~ns}$ the transition time of the electronics between the two logical states becomes evident as a cutoff in the distribution. For intervals of up to $35 \mathrm{~ns}$ some wiggles of the distribution are apparent. This is most likely due to ringing of the signals on the transmission line. For times $>35$ ns the distribution approaches an exponential decay function. The spike at 96 ns was identified as an artifact of the counter due to an internal 
time marker.

As described earlier, our device can produce random numbers by periodically sampling the signal and cyclically transferring the data to a personal computer. Our personal computer (Pentium processor, $120 \mathrm{MHz}, 144 \mathrm{MB}$ RAM, running LabView on Win95) manages to register sets of random numbers up to a size of 15 MByte in a single run at a maximum sample rate of $30 \mathrm{MHz}$. In order to obtain independent and evenly distributed random numbers, the sampling period must be well above the autocorrelation time of the random signal. We observed that for a signal autocorrelation time of roughly $20 \mathrm{~ns}$ a sampling rate of $1 \mathrm{MHz}$ suffices for obtaining "good" random numbers.

All data samples used for the following evaluations consisted of $80 \cdot 10^{6}$ bits produced in continuous runs with a $1 \mathrm{MHz}$ bit sampling frequency. Figure 6(a) depicts the distribution of blocks with 8 bit length within a data sample. This distribution approaches an even distribution, but still shows some non-statistical deviations, such as a peak at in the center and some symmetric deviations. Possibly this is due to a yet to high sampling rate and a slight misadjustment of the generator. The distribution of blocks of $n$ concatenated zeros and ones within a sample should be proportional to a $2^{-n}$-function. (Figure 6(b)) The slopes of the logarithmically scaled distributions are measured with a linear fit and are $-0,29725 \pm 0,00121$ for the $n$-zero blocks and $-0.30299 \pm 0.00138$ in the case of the $n$-one blocks. Ideally, the slopes should both be equal to $-\log (2)=-0,30103$. The deviation can be understood as a consequence of minor differences in the probabilities of finding a zero or a one at the output of the generator, again due to misadjustment of the generator.

The mean value of 8-bit blocks, the entropy for 8-bit blocks and the Monte Carlo estimation of $\pi$ are evaluated for a data sample produced by our random generator and compared to data samples taken from the Marsaglia $\mathrm{CD}-\mathrm{ROM} 3$ and a sample data set built with the Turbo $\mathrm{C}++$ random function 11 . (Table 【)

The results in Table $\mathbb{I}$ are in favor of our device but the numbers must be treated with caution, as they represent only a comparison of single samples which may not be representative. 


\section{DISCUSSION AND OUTLOOK}

The experimental results presented in the chapter above gives strong support to the expectation, that our physical quantum random generator is capable of producing a random binary sequence with an autocorrelation time of $12 \mathrm{~ns}$ and internal delay time of $75 \mathrm{~ns}$. This underlines the suitability of these devices for their use in our specific experiment demanding random signal generators with a time for establishing a random output state to be less than $<100$ ns, which is easily achieved by the physical quantum random generators presented in this paper.

The high speed of our random generators is made possible by the implementation of state of the art technology using fast single photon detectors as well as high speed electronics. Moreover, the collection of tests applied to the signals and random numbers produced with our quantum random generator demonstrate the quality of randomness that is obtained by using a fundamental quantum mechanical decision as a source of randomness.

Some methods for enhancing the performance, be it in terms of signal equidistribution and/or autocorrelation time, can be foreseen. For instance, a different method for generating the random signal would be that each of the PM's toggles a $\frac{1}{2}$-divider which results in evenly distributed signals. These signals could be combined in an XOR-gate in order to utilize the quantum randomness of the polarization analyzer, but fully keeping the equidistribution of the signal. A reduction of the signal autocorrelation time is possible by optimizing the signal electronics for speed (e.g. using ECL signals throughout the design). Further, it is simple to parallelize several such random generators within one single device, as there is no crosstalk between the subunits, since the elementary quantum mechanical processes are completely independent and undetermined. Hence designing a physical quantum random number generator capable of producing true random numbers at rates $>100 \mathrm{MBit} / \mathrm{s}$ or even above $1 \mathrm{GBit} / \mathrm{s}$ is a feasible task22.

We believe that random generators designed around elementary quantum mechanical processes will eventually find many applications for the production of random signals and 
numbers, since the source of randomness is clear and the devices operate in a straightforward fashion.

\section{ACKNOWLEDGEMENT}

This work was supported by the Austrian Science Foundation (FWF), project S6502, by the U.S. NSF grant no. PHY 97-22614, and by the APART program of the Austrian Academy of Sciences. 


\section{REFERENCES}

${ }^{1}$ J. Alspector, B. Gupta, and R. B. Allen, Adv. Neural Inform. Proc. Syst. 1, 748 (1989).

${ }^{2}$ G. Marsaglia, Computers \& Mathematics with Applications, 9, 1 (1993).

${ }^{3}$ G. Marsaglia, The Marsaglia Random Number CDROM, Department of Statistics and Supercomputer Computations Research Institute, Florida Sate University (1995).

${ }^{4}$ U. Maurer, Proceedings of CRYPTO '90, Santa Barbara CA (1990).

${ }^{5}$ C. M. Ellison, published on the internet: www.clark.net/pub/cme/P1363/ranno.html (1995).

${ }^{6}$ For a review see: C. H. Bennett et al., Scientific American 257(10), 50 (1992).

${ }^{7}$ G. Weihs, T. Jennewein, C. Simon, H. Weinfurter, and A. Zeilinger, Phys. Rev. Lett. 81, 5039 (1998).

${ }^{8}$ Matsumoto, Kurita, ACM Transactions on Modelling and Computer Simulation 4(3), 254 (1997).

${ }^{9}$ M. Isida, H. Ikeda, Ann. Inst. Stat. Math. Tokyo 8, 119 (1956).

${ }^{10} \mathrm{~J}$. Walker, published on the internet: www.fourmilab.ch/hotbits/how.htm (1996).

${ }^{11}$ U. Achleitner, Diploma Thesis, Innsbruck University (1997).

12 A. J. Martino, G. M. Morris, Applied Optics 30, 981 (1991).

${ }^{13}$ G. M. Morris, Opt. Engin. 24, 86 (1985); J. Marron, A. J. Martino, G. M. Morris, Applied Optics 25, 26 (1986).

${ }^{14}$ W. M. Itano, J. C. Bergquist, R. G. Hulet, and D. J. Wineland, Phys. Rev. Lett. 59, 2732 (1987).

15 Th. Sauter, W. Neuhauser, R. Blatt, and P. E. Toschek, Phys. Rev. Lett. 57, 1696 (1986). 
${ }^{16}$ Photomultiplier module, type HP5783-P, selected for single photon detection, Hamamatsu Photonics Deutschland GmbH, D-82211 Herrsching am Ammersee, Germany.

17 A. Compagner, Am. J. Phys. 59, 700 (1991).

${ }^{18}$ V. A. Uspekhi, A. L. Semenov, A. K. Shen, Russian Math. Surveys 45(1), 121 (1990).

${ }^{19}$ P. L'Ecuyer, Proceedings of the 1992 Winter Simulation Conference, 305, IEEE Press (1992).

${ }^{20}$ L. Vattulainen, T. Ala-Nissila, Computers in Physics 9, 500 (1995).

${ }^{21}$ See e.g.: L. Mandel and E. Wolf, Cambridge University Press (1995).

${ }^{22}$ P. Lalanne et al., Opt. Engin. 32, 1904 (1993). 


\section{FIGURES}

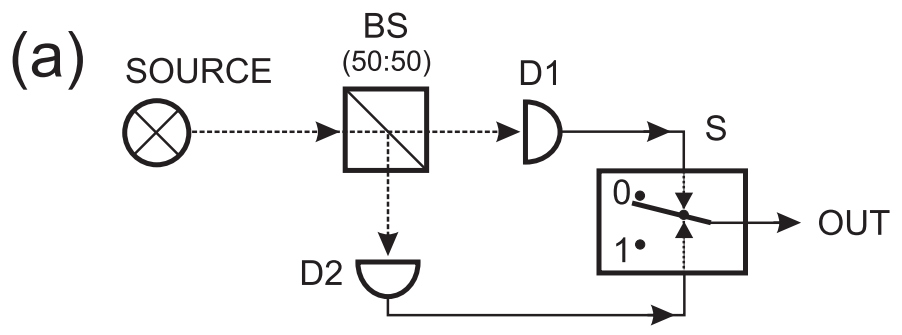

(b)

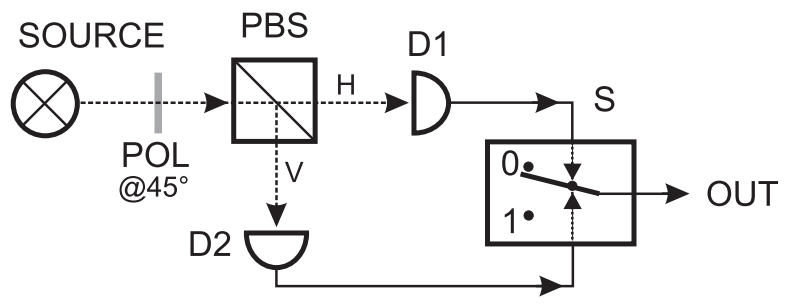

(c)

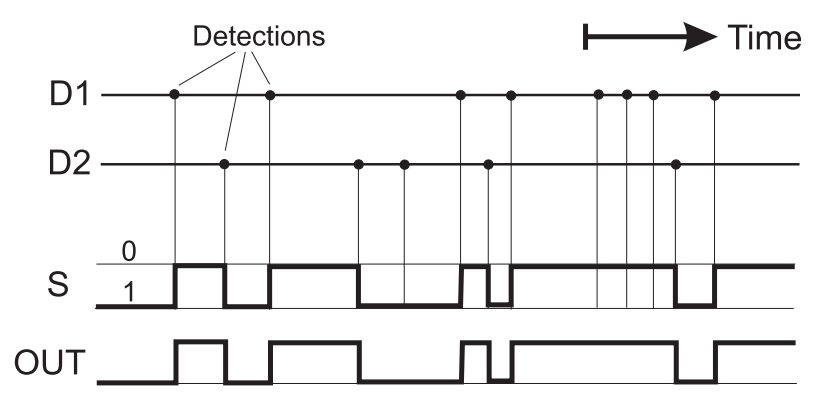

FIG. 1. The source of randomness within our device is the splitting of a weak light beam. This is realized with a 50:50 optical beam splitter BS (a) or a polarizing beam splitter PBS where the incoming light is polarized with POL at $45^{\circ}$ with respect to the PBS (b). The photon detections of the detectors D1 and D2 in the two output paths toggle the switch S between its two states (c). This produces a randomly alternating binary signal OUT. 


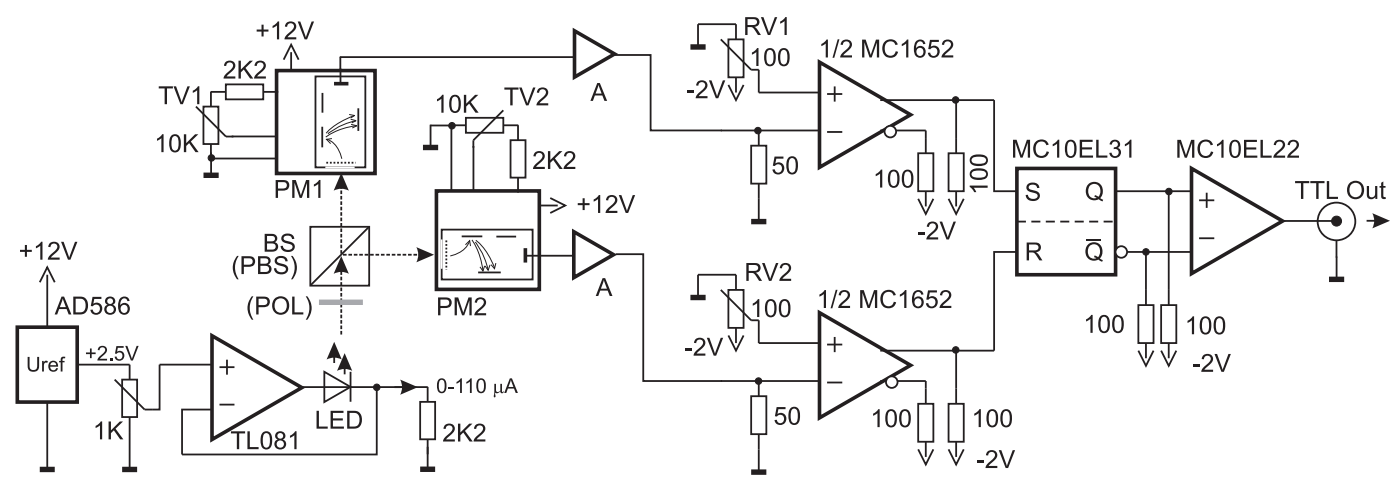

FIG. 2. Circuit diagram of the physical quantum random generator. To the left is the light source (LED) and the configuration of the beam splitter (BS/PBS) and the two photo multipliers (PM1, PM2). The detection pulses of the PMs are turned into standard logic pulses with discriminators (MC1652) and combined in the RS-flip-flop (MC10EL31) to generate the random signal.

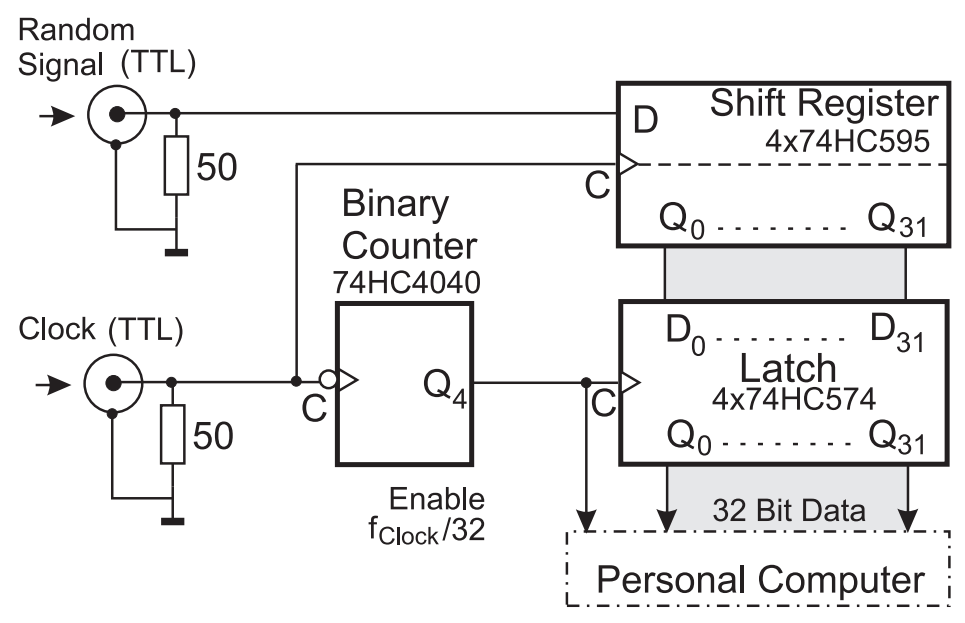

FIG. 3. Schematic diagram of the circuit for transferring random numbers to a personal computer at a constant bit rate. 


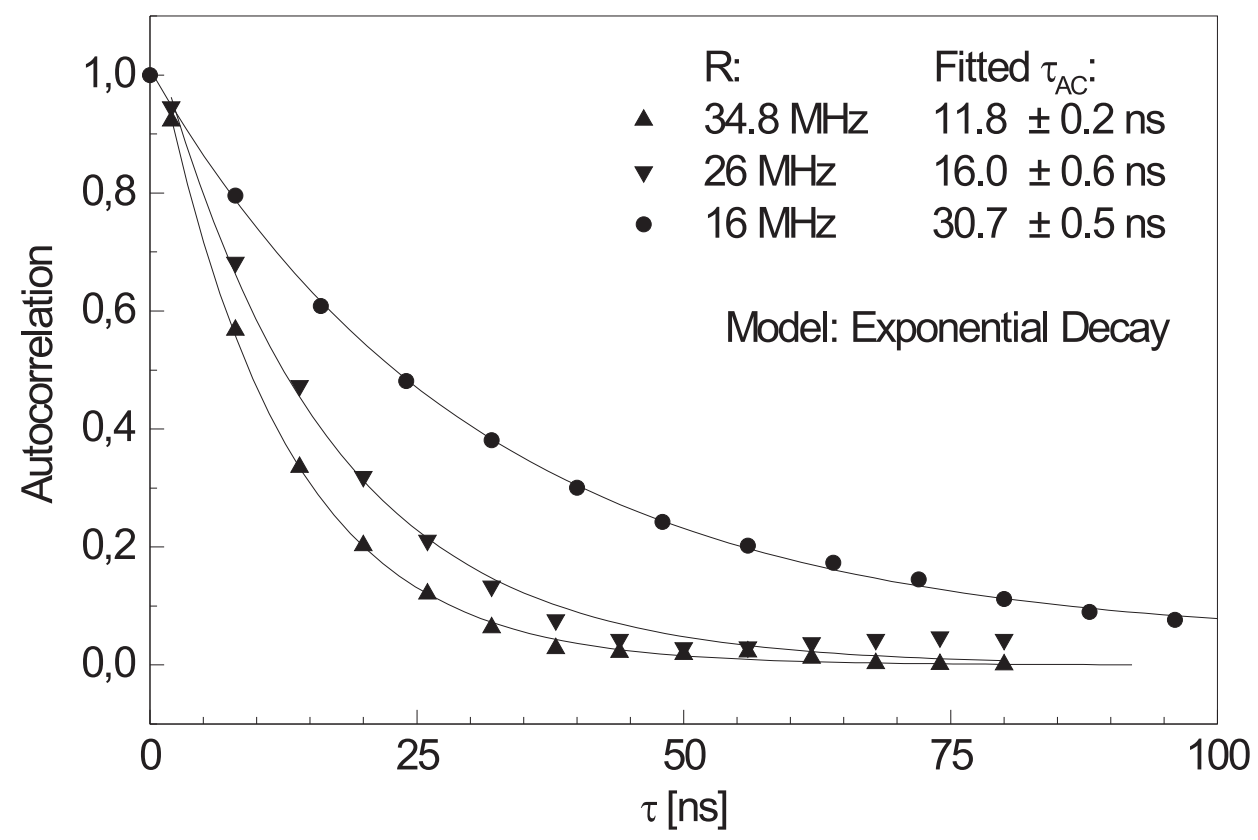

FIG. 4. The autocorrelation functions computed from traces of the random signal with different average signal toggle rates. The given autocorrelation times are obtained with an exponential decay fit. 


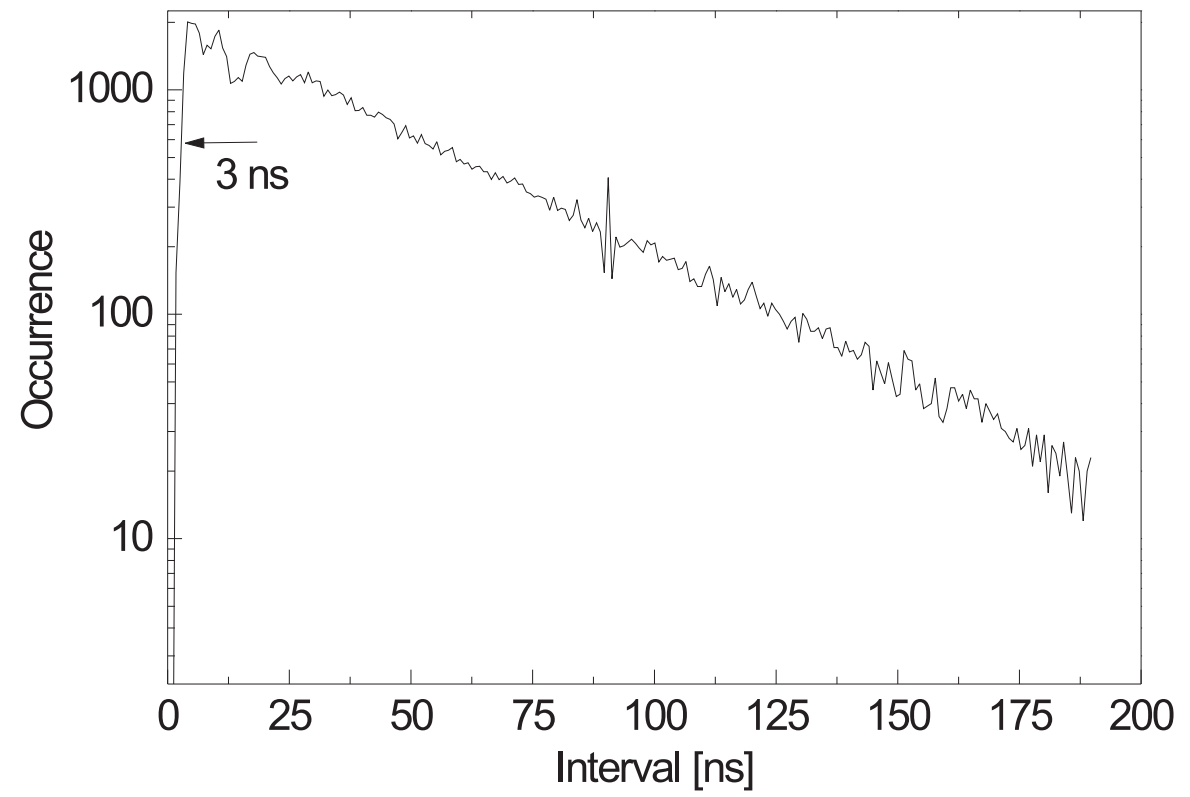

FIG. 5. Distribution of $10^{6}$ time intervals between successive transitions of the random signal with an average toggle rate of $26 \mathrm{MHz}$. This distribution follows an exponential decay function for times $>35 \mathrm{~ns}$. (The spike at $96 \mathrm{~ns}$ is from the counter itself.) 

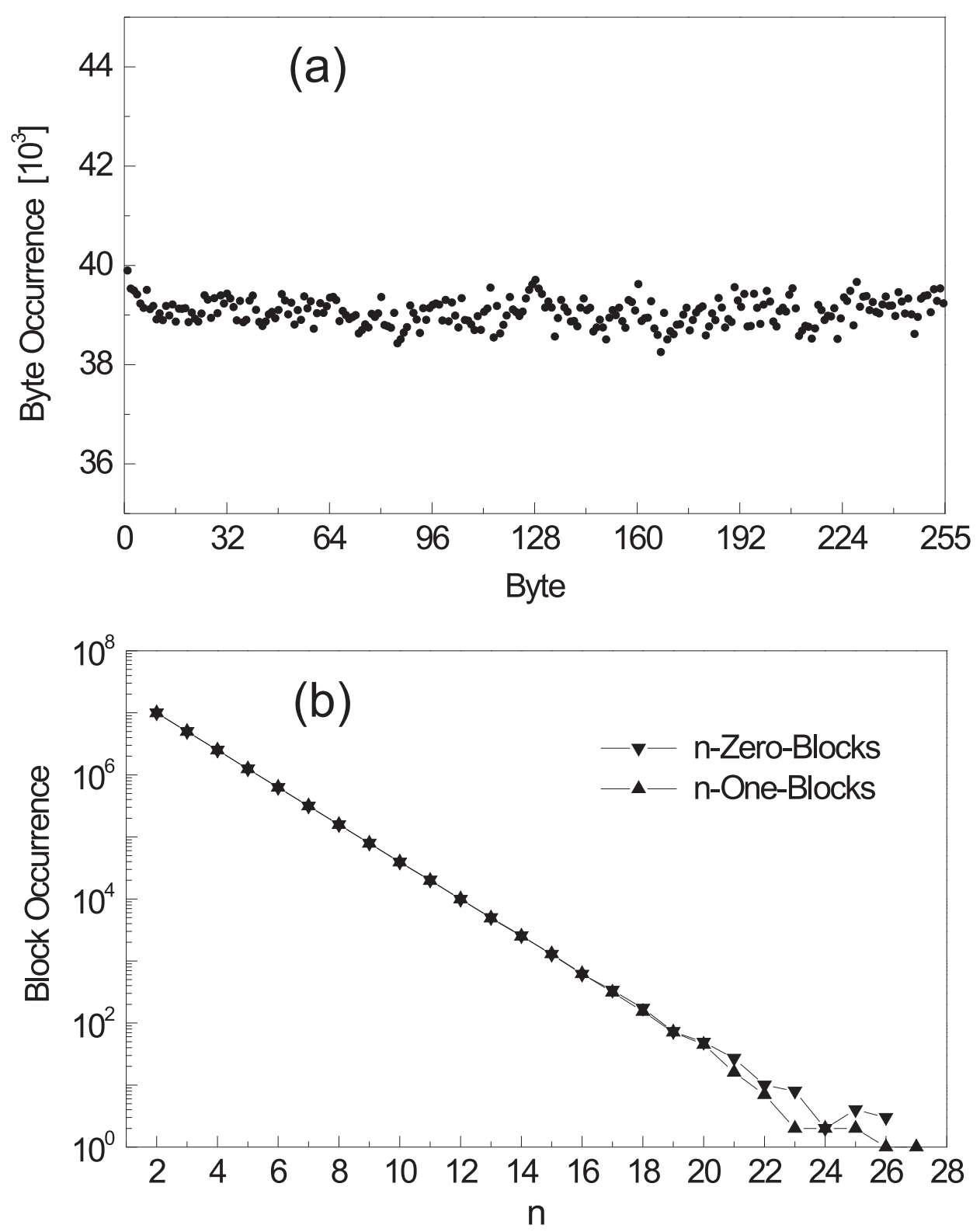

FIG. 6. Diagram (a): distribution of bytes within $80 \cdot 10^{6}$ random bits produced with the random number generator. Diagram (b): the occurrence of blocks of concatenated zeros and ones within the same set of random numbers. 


\section{TABLES}

\begin{tabular}{c|r|r|r|r}
\hline \hline & QRNG & Bits52 & Canada & C++ \\
\hline \hline Mean & 127.50 & 127.50 & 126.58 & 127.22 \\
Entropy & 7.999965 & 7.999982 & 7.997665 & 7.81118 \\
$\pi$ & 3.14017 & 3.14367 & 3.15789 & 3.15761 \\
\hline \hline
\end{tabular}

TABLE I. Evaluation of tests of randomness for data samples taken from a selection of sources. QRNG: data set generated with our physical quantum random generator, Bits52: taken from the Marsaglia CD-ROMB, data set generated with a combination of pseudo random generators, Canada: taken from the Marsaglia CD-ROM, data set generated with a commercial physical random generator, $\mathrm{C}++$ : data set generated with the pseudo random generator within Turbo $\mathrm{C}++$ (Borland Inc.) 11 . 